\title{
circ_ZFR Is Linked to Paclitaxel Resistance in Cervical Cancer via miR-944 Sponging and IL-10 Upregulation
}

\author{
Xiaoqian Long, Meiyun Zheng, Youlin Yang, Yi Chen, Xiahui Zhang, and Haiyan Zhang \\ Department of Obstetrics and Gynecology, The First People's Hospital of Wenling, Wenling, 317599 Zhejiang, China \\ Correspondence should be addressed to Haiyan Zhang; zhanghaiyanwz@126.com
}

Received 10 November 2021; Accepted 29 December 2021; Published 27 January 2022

Academic Editor: Giuseppe Broggi

Copyright (C) 2022 Xiaoqian Long et al. This is an open access article distributed under the Creative Commons Attribution License, which permits unrestricted use, distribution, and reproduction in any medium, provided the original work is properly cited.

\begin{abstract}
Objective. Cervical cancer (CC) has an elevated rate of invasion and death despite surgical treatment, radiotherapy, and chemotherapy. Several studies revealed that circRNAs have a key contribution to the resistance of drugs against different types of carcinomas. The goal of the existing study was to figure out what role circ_ZFR plays in paclitaxel (PTX) resistance in cervical cancer (CC) patients. Materials and Methods. Herein, two types of CC cells (SiHa/PTX and Hela/PTX) were utilized. The levels of IL-10 mRNA, miR-944, and circ_ZFR were measured using qRT-PCR analyses. The CCK-8 assay was used to determine PTX resistance. The IL-10 expression was measured via the ELISA technique. The combination of miR-944 and circ_ZFR or IL-10 was validated using a dual-luciferase reporter (DLR) assay. Results. The amount of circ_ZFR was increased in PTX-resistant CC cells and tissues. In PTX-resistant CC cells, knocking down circ_ZFR expression decreased PTX resistance. circ_ZFR knockdown significantly reduced IL-10 expression via sponging miR-944, increasing PTX sensitivity in PTX-resistant CC cells. Conclusion. circ_ZFR knockdown has a considerable role in overwhelming CC-associated PTX resistance by modifying the axis of miR-944/IL-10 axis, suggesting that developing a circRNA target-based treatment could be considered prevent CC progression.
\end{abstract}

\section{Introduction}

According to the global survey of cancer statistics, cervical cancer (CC) is considered to be the fourth most prevalent malignancy in women despite the HPV (human papillomavirus) vaccination and testing $[1,2]$. CC has an elevated rate of invasion and death despite surgical treatment, radiotherapy, and chemotherapy. For resistant or recurring CC, the antitumor drug paclitaxel (PTX) is now commonly utilized, but PTX resistance has a significant impact on its therapeutic benefit [3]. As a result, enhancing clinical treatment methods requires extensive knowledge of the molecular mechanisms underlying PTX resistance in CC.

circRNAs are a type of unique noncoding RNA $[4,5]$ without $3^{\prime}$ and $5^{\prime}$ ends. Both ends are covalently connected which provides a circular shape to the RNA molecule [6,7]. circRNAs were originally considered as splicing defects [8]; however, numerous studies have shown that these RNAs with abnormal expression have a key role in the advancement of various malignancies [9-12], including CC $[13,14]$. circRNAs have been shown to influence chemoresistance [15-17]. circ_ZFR (also known as hsa-circ 0072088) promotes CC progression by $\mathrm{Rb}$ phosphorylation and through regulating the SSBP1/ CDK2/cyclin E1 complex [18]. However, the implications of circ_ZFR for the CC's PTX resistance are remaining elusive.

The critical role of the circRNA-miRNA-mRNA regulation network in chemoresistance has been gradually explored [19-21]. According to our obtained data, miR944 has the same binding sequences as circ_ZFR and IL-10 in the existing study, suggesting that circ_ZFR could act as a sponge for miR-944 to boost IL-10 expression. As a result, we decided to evaluate the implications of the circ_ZFR/ miR-944/IL-10 axis for CC PTX resistance.

\section{Materials and Methods}

2.1. Tissue Acquisition. At Wenling First People's Hospital, 96 CC tissue samples (62 cervical squamous cell carcinoma 


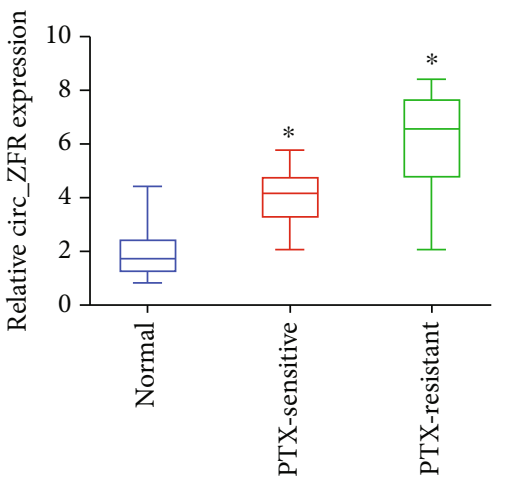

(a)

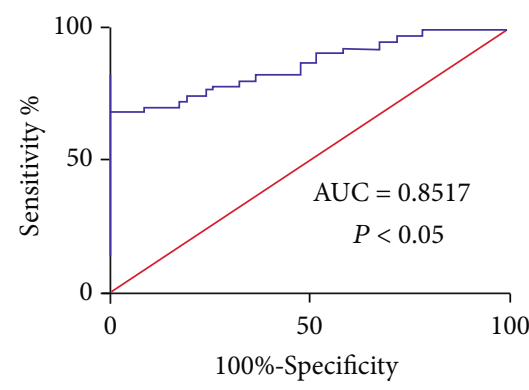

(c)

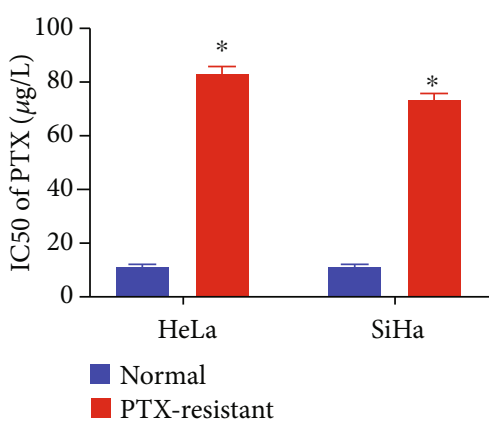

(e)

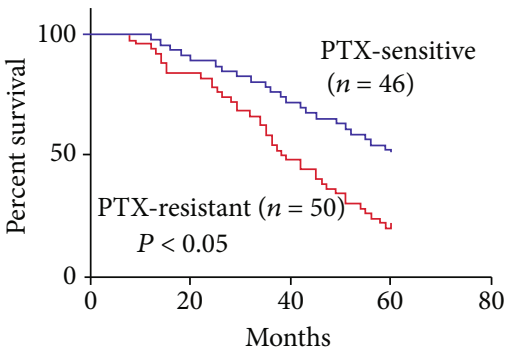

(b)

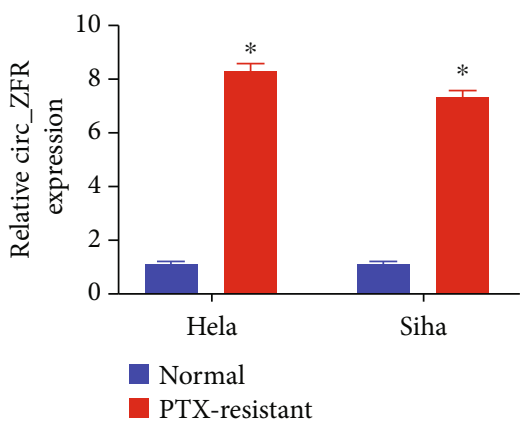

(d)

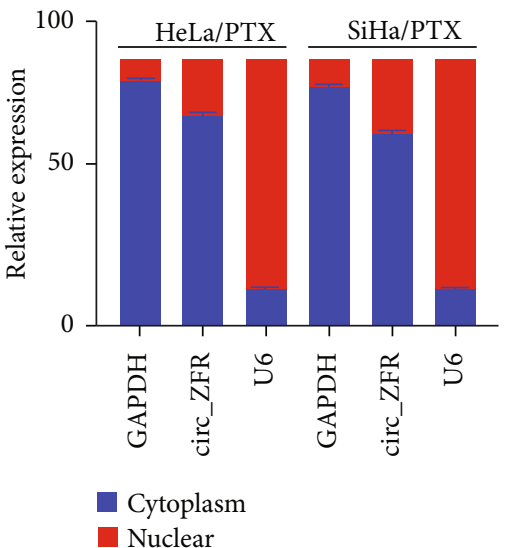

(f)

FIgURE 1: The overexpression of circ_ZFR in CC cells and tissues (PTX-resistant). (a) The qRT-PCR evaluations of circ_ZFR expression in CC tissues. (b) The survival rate in CC patients. (c) The ROC curve analysis of circ_ZFR for CC resistance. (d) The data of circ_ZFR expression obtained from the qRT-PCR assay. (e) The PTX IC I0 $_{50}$ values. (f) circ_ZFR expression in the nuclear and cytoplasm with ${ }^{*} p<0.05$.

and 34 cervical adenocarcinoma) and surrounding healthy tissue specimens were taken from patients associated with CC, followed by classification into two groups, i.e., the treatment-resistant group $(n=50)$ and the treatmentsensitive $(n=46)$ group. The existing study was authorized by the Wenling First People's Hospital Ethics Committee. All of the patients who took part in this study signed a written informed consent form.

2.2. Cell Proliferation and Cell Transfection. Human CC cell lines ( $\mathrm{SiHa}$ and $\mathrm{HeLa}$ ) were provided by the Shanghai Institutes for Biological Sciences (SIBS), China. The underlined cell was grown in liquid HyClone DMEM (Logan,
UT, USA) along with 10\% FBS. The culture mixtures were incubated $\left(\right.$ at $\left.37^{\circ} \mathrm{C}\right)$ in the presence of air enriched with $5 \% \mathrm{CO}_{2}$. HeLa and $\mathrm{SiHa}$ cell lines were cultured with the concentration of PTX increased from 10 to $20 \mu \mathrm{g} / \mathrm{L}$ and $40 \mu \mathrm{g} / \mathrm{L}$. After 10 months of HeLa and SiHa cell lines' continuous culturing, PTX-resistant Hela/PTX and SiHa/ PTX cell lines could be collected which maintained a good growth state in $40 \mu \mathrm{g} / \mathrm{L}$ PTX. The SiHa/PTX and Hela/ PTX cells were seeded in 6-well plates and incubated for $24 \mathrm{hrs}$ before being treated with $50 \mathrm{nM}$ of appropriate synthetic oligonucleotides or victor transfection $(2 \mu \mathrm{g})$ using Lipofectamine 2000 (11668500; Invitrogen), as the manufacturer recommended. 


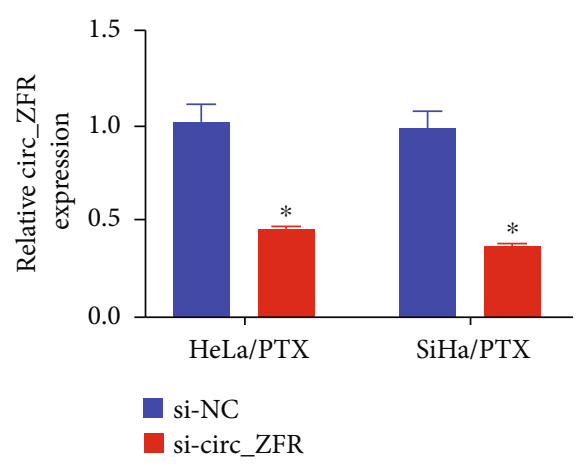

(a)

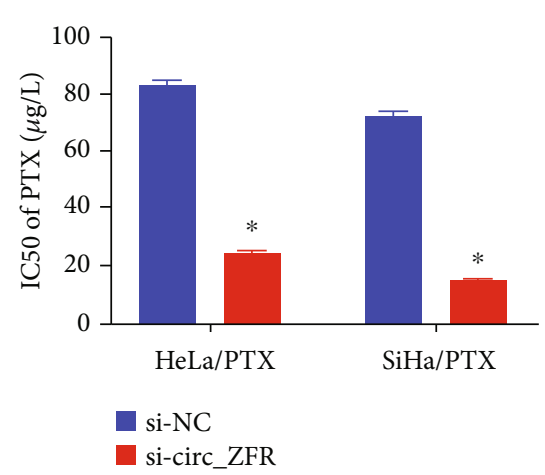

(b)

FIGURE 2: The suppression level of PTX resistance in circ_ZFR knockdown PTX-resistant CC cells. (a) The level of circ_ZFR expression in $\mathrm{SiHa} / \mathrm{PTX}$ and HeLa/PTX cells. (b) The $\mathrm{IC}_{50}$ values of PTX for SiHa/PTX and HeLa/PTX cells with ${ }^{*} p<0.05$.

2.3. $q R T-P C R$ Analyses. Following the manufacturer's recommendation, TRIzol (Invitrogen) was utilized to obtain total-RNA from CC cells or tissues. The TaqMan miRNA experiment was carried out to assess the amount of miR944 (Thermo Fisher Sci, Inc., Waltham, MA, USA). The cDNA was extracted from $1 \mu \mathrm{g}$ of obtained RNA to evaluate the expression level of IL-10 and circ_ZFR by employing the Prime-Script-RT reagent Kit with gDNA remover (Takara, Dalian, China). $10 \mu \mathrm{L}$ of Go-Taq-qPCR Master Mix (Promega, Madison, WI, USA) was used to perform qRT-PCR analyses. In the existing study, we used U6 and GAPDH as reference genes. The $2^{-\Delta \Delta \mathrm{CT}}$ approach was followed to analyze the obtained data. To assess the miR-944 expression, reverse transcription was carried out via a particular stemloop RT-primer: 5 '-CTCAACTGGTGTCGTGGAGTC GGCAATTCAGTTGAGCTCATCCG-3'.

The nucleotide sequences of the primer against circ_ ZFR, IL-10, U6, and GAPDH have been shown as follows: circ_ZFR forward (F), $5^{\prime}$-AACCACCACAGATTCACTAT$3^{\prime}$ and reverse (R), 5' -AACCACCACAGATTCACTAT-3'; IL-10 F, $5^{\prime}$-GCCAAGCCTTGTCTGAGATGATCC- $3^{\prime}$ and R, $5^{\prime}$-TTCACATGCGCCTTGATGTCTGG-3'; U6 F, $5^{\prime}$ TGCGGGTGCTCGCTTCGGCAGC-3' and R, $5^{\prime}$-CCAG TGCAGGGTCCGAGGT-3'; and GAPDH F, $5^{\prime}$-CATGAG AAGTATGACAACAGCCT- $3^{\prime}$ and $\mathrm{R}, 5^{\prime}$-AGTCCTTCC ACGATACCAAAGT-3'.

2.4. PTX $I C_{50}$ Assay. Using 96-well plates, $2 \times 10^{3}$ transfected Hela/PTX and SiHa/PTX cells were grown and were then treated with different PTX concentrations $(0.1,1,10$, 100 , and $1000 \mu \mathrm{g} / \mathrm{L})$. After $48 \mathrm{hrs}$, the seeded cells were treated with the CCK-8 solution $(10 \mu \mathrm{L})$, followed by thoroughly mixing them for $2 \mathrm{hrs}$. After the treatment of the CCK-8 solution, the O.D. (at $450 \mathrm{~nm}$ ) of each well was recorded via an automated Multiskan-mk3 microplate reader (Thermo Fisher, USA).

2.5. Dual-Luciferase Reporter (DLR) Assay. The complete or mutant segments of the circ_ZFR or IL-10 3'UTR comprising the expected miR-944 interacting sites were cloned downstream of the luciferase gene in the pGL3 vector (Pro- mega, Fitchburg, WI, USA). The cotransfection of 293 T cells was carried out with miR-NC or miR-944 in combination with IL-10 $3^{\prime}$ UTR-MUT or IL-10 $3^{\prime}$ UTR-WT or circ ZFR-MUT or circ_ZFR-WT. After $24 \mathrm{hrs}$ of the incubation period, luciferase activity was assessed using the DLR assay.

2.6. RNA Immunoprecipitation (RIP) Assay. The RIP assay was carried out with the help of the Magna RIP kit (Millipore). On the basis provided instructions (user's manual), Hela/PTX and $\mathrm{SiHa} / \mathrm{PTX}$ cell transfection was carried out with miR-944 or miR-NC and cultured for 48 hrs before being lysed with RIP buffer having IgG-antibody or Ago2-labeled magnetic beads. Following that, TRIzol reagent was utilized to obtain immunoprecipitated RNAs. Furthermore, the circ_ZFR enrichment was determined via qRT-PCR evaluations.

2.7. RNA Pull-Down Assay. SiHa/PTX and Hela/PTX cell transfection was carried out via biotinylated circ_ZFRMUT or ZFR-WT or NC, followed by $48 \mathrm{hrs}$ of incubation. The cells were collected, lysed with RIP buffer, and then treated with streptavidin magnetic beads (M-280; Invitrogen) according to the manufacturer's instructions. The beads were thricely rinsed via a cold buffer after being incubated in a cold chamber $\left(4^{\circ} \mathrm{C}\right)$ for two hours. Eventually, the RNA elution was carried out so that it could be used in other experimental evaluations.

2.8. ELISA. Following the instructions of the provider, an ELISA kit ( $\mathrm{R} \& \mathrm{D}$ Systems) was employed for the measurement of IL-10 expression in various culture media. The average value was calculated from three experiments evaluated separately.

2.9. Statistics Analysis. The obtained data was compiled using SD from triplicated expressions (individually performed). GraphPad Ver 7.04 (La Jolla, CA, USA) was employed for the statistical evaluations on the data obtained. The means of continuous outcome variables were examined via ANOVA. Kaplan-Meier plots and log-rank test were conducted for the analysis of survival rates and to investigate variations among subgroups of patients. Variations were regarded as statistically considerable when the $p$ values were less than 0.05 . 
Circ_ZFR-WT 5'-ugUAUCUGAAGCGGCAAUAAUUu-3’ || || || | miR-944 3'-gaGUAGGCUACAUGUUAUUAAa-5' Circ_ZFR-MUT 5'-ugUAUCUGAAGCGGGUUAUUAAu-3'

(a)

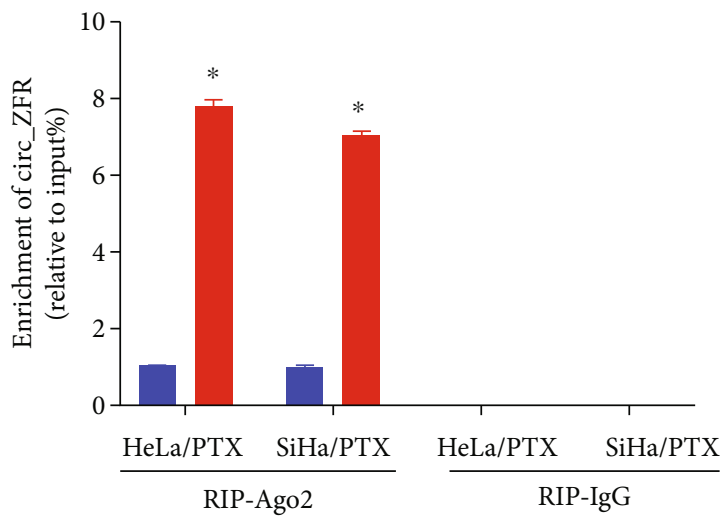

a miR-NC

miR-944

(c)

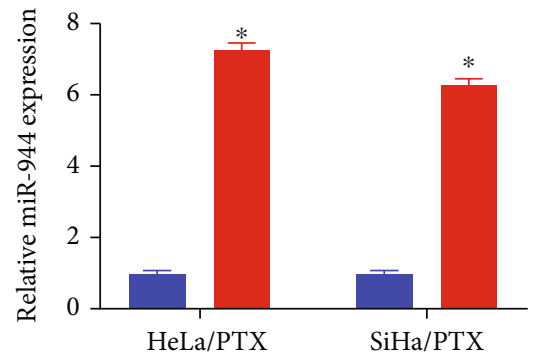

si-NC

a si-circ_ZFR

(e)

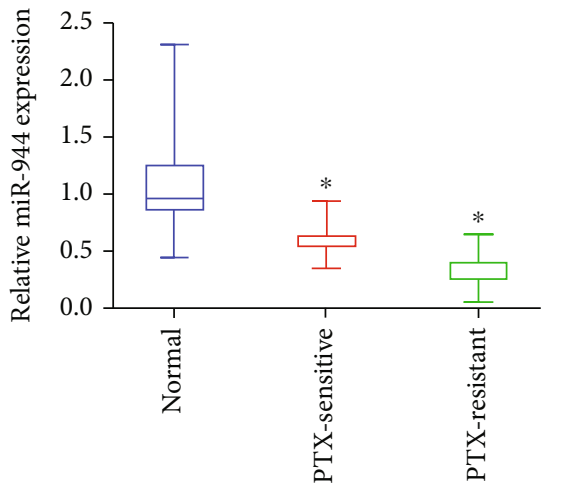

(g)

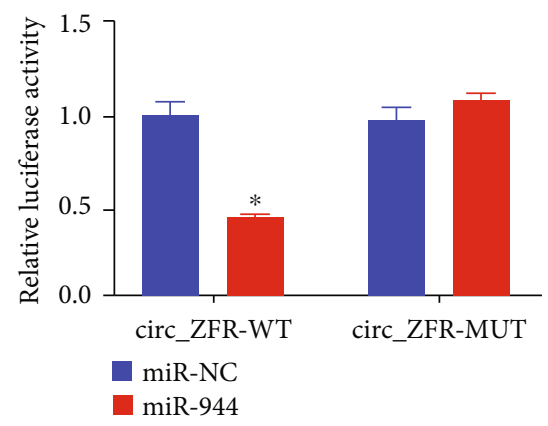

(b)

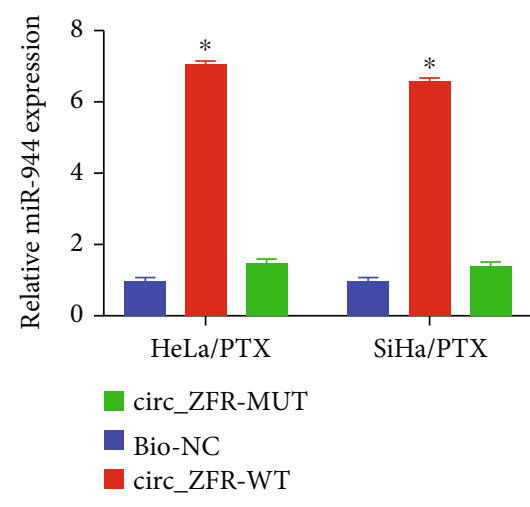

(d)

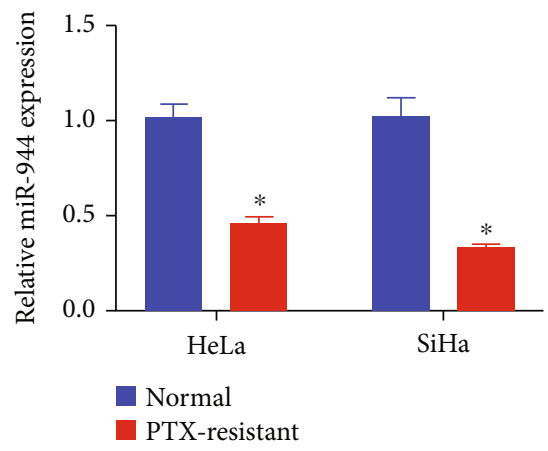

(f)

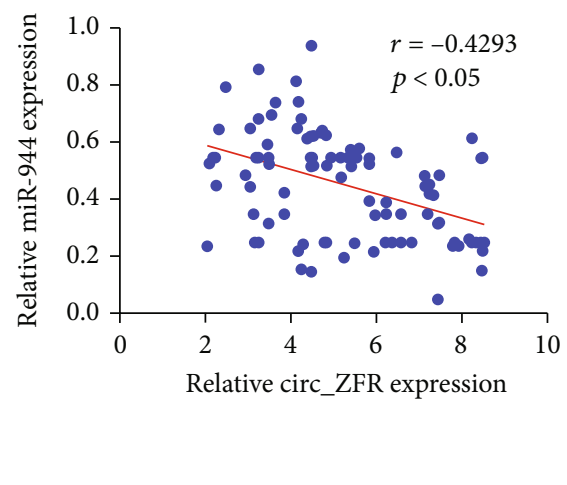

(h)

FIgURe 3: Continued. 


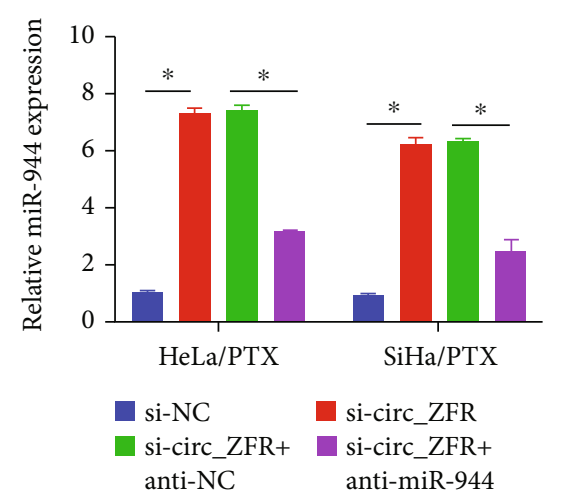

(i)

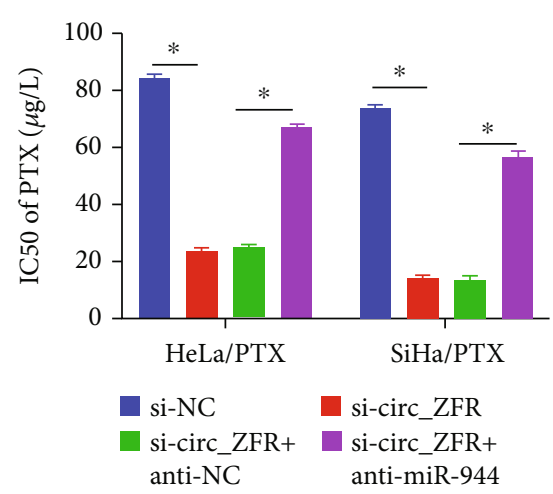

(j)

FIgURE 3: The miR-944 inhibition reduced the impact of circ_ZFR knockdown in PTX-resistant CC cells. (a) The possible interacting domains for miR-944 and circ_ZFR. (b) The interaction between miR-944 and circ_ZFR evaluated by DLR assay. (c, d) The data obtained from RIP and RNA pull-down assays accordingly. (e, f) The qRT-PCR data to evaluate the expression of miR-944. (g) The miR-944 expression in CC tissues. (h) The linkage between miR-944 and circ_ZFR. (i) The miR-944 expression level in SiHa/PTX and HeLa/PTX cell lines. (j) The PTX IC 50 values in SiHa/PTX and HeLa/PTX cells. ${ }^{*} p<0.05$.

\section{Results}

3.1. An Elevated Expression of circ_ZFR in CC Tissues and Cell Lines (Resistant to PTX). The circ_ZFR expression was evaluated in PTX-sensitive and PTX-resistant CC tissues via qRT-PCR analysis. The obtained data suggested that circ_ZFR expression was found to be elevated in CC tissues (resistant to PTX) in comparison with the healthy tissues and CC tissues (sensitive to PTX), as represented in Figure 1(a). Patients who were PTX-sensitive had a significant survival rate when treated with PTX, as indicated in Figure 1(b). The results of ROC curves showed that circ_ ZFR may have diagnostic significance for PTX-resistant CC patients (Figure $1(\mathrm{c})$ ). Furthermore, in HeLa and SiHa cells, the level of circ_ZFR was decreased relative to HeLa/ PTX and SiHa/PTX cells, as depicted in Figure 1(d). The results obtained from the CCK- 8 assay showed PTX resistance in HeLa/PTX and SiHa/PTX cells, as revealed by the higher $\mathrm{IC}_{50}$ concentration of PTX in HeLa/PTX and SiHa/ PTX cells, as depicted in Figure 1(e). circ_ZFR was mostly found in the cytoplasmic part of the underlined cells, as indicated in Figure 1(f). The underlined data revealed that dysregulated circ_ZFR may have a role in the chemoresistance of CC.

3.2. Suppression of PTX Resistance in CC Cells (Resistant to PTX) by circ_ZFR Knockdown. To determine the role of circ_ZFR in PTX-resistant CC cells, si-circ_ZFR was transfected into HeLa/PTX and SiHa/PTX cells to knock down the circ_ZFR expression, and relative to si-NC groups, a considerable decrease was observed in the expression of circ_ZFR in HeLa/PTX and SiHa/PTX cells, as suggested by the qRT-PCR (Figure 2(a)). PTX IC $_{50}$ value was lowered in the underlined cells (transfected with si-circ_ ZFR), as indicated by CCK-8 assay which suggested that circ_ZFR knockdown overcomes PTX resistance in the underlined cells, as depicted in Figure 2(b). In PTXresistant CC cells, knocking down circ_ZFR boosted the sensitivity of PTX.
3.3. Attenuation of miR-944 Enhanced the Impact of circ ZFR Knockdown on PTX Sensitivity in CC Cells (Resistant to PTX). Online tool starbase 3.0 was employed to examine the possible target of circ_ZFR to explore the pathway associated with circ_ZFR to regulate PTX resistance and cellular progression in CC cells (PTX resistant). miR-195-5p could be a circ_ZFR target, as it had the binding sites of circ ZFR (Figure 3(a)). The DLR assay revealed that miR-994 transfection significantly reduced circ_ZFR-WT luciferase activity while not affecting circ_ZFR-MUT luciferase activity in $293 \mathrm{~T}$ cells, validating the link between circ_ZFR and miR-944, as depicted in Figure 3(b). Anti-AGO2 antibody was used to enrich endogenous circ_ZFR (Figure 3(c)). In HeLa/PTX and SiHa/PTX cells, the wild-type circ_ZFR collected more miR-944 than the mutant revealed by RNA pulldown assay (Figure 3(d)), showing that circ_ZFR directly targets miR-944. Furthermore, we discovered that silencing circ_ZFR increased the expression of miR-944 in the underlined cells, as depicted in Figure 3(e). The expression of miR944 was elevated in HeLa and $\mathrm{SiHa}$ cells relative to HeLa/ PTX and SiHa/PTX cells, as depicted in Figure 3(f). Furthermore, the miR-944 level decreased from normal tissues to CC tissues (PTX-sensitive) and then to CC tissues (PTXresistant), as indicated in Figure $3(\mathrm{~g})$. It has also been depicted that there was a negative association, existed between miR-944 and the circ_ZFR expression in tumor tissues (Figure 3(h)). miR-944 was negatively regulated by circ_ZFR through directly targeting. Based on the above findings, rescue experiments were conducted to evaluate whether PTX resistance has been regulated by circ_ZFR through miR-944 targeting. An elevated expression of miR944 (inducted by si-circ_ZFR) was found to be reversed via anti-miR-944 transfection in HeLa/PTX and SiHa/PTX cells, as indicated in Figure 3(i). By lowering miR-944, the inhibitory effects of circ_ZFR knockdown on PTX resistance were all reversed in HeLa/PTX and SiHa/PTX cells (Figure 3(j)). The underlined results indicated that through sponging miR-944, circ_ZFR knockdown suppressed PTX-resistant CC cells. 


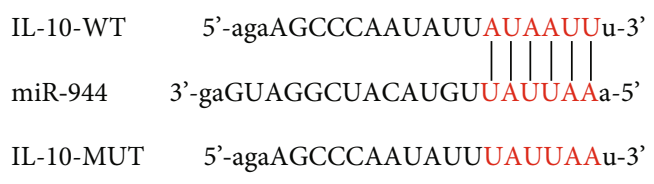

(a)

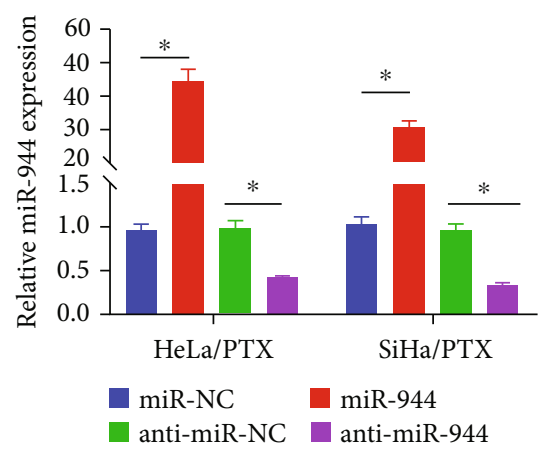

(c)

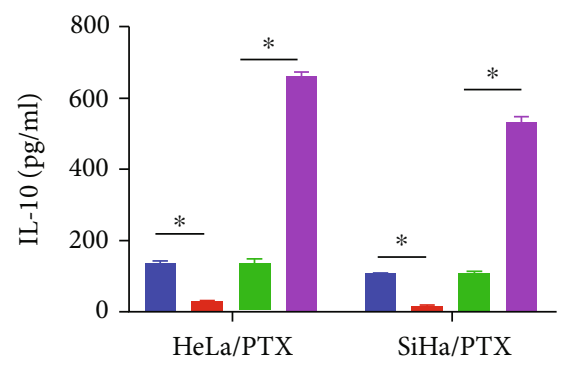

miR-NC

anti-miR-NC anti-miR-944

(e)

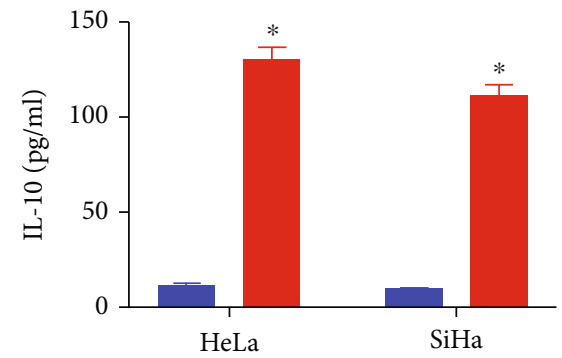

normal

PTX-resistant

(g)

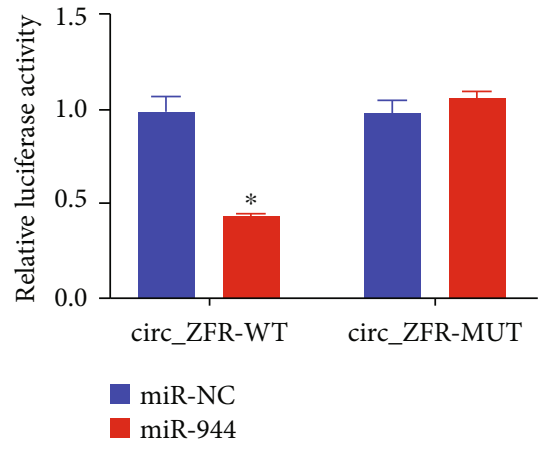

(b)

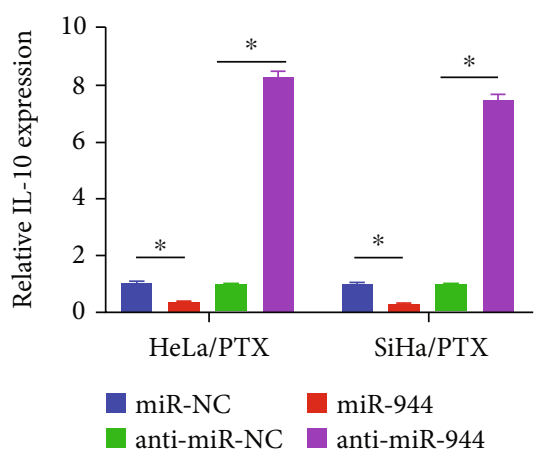

(d)

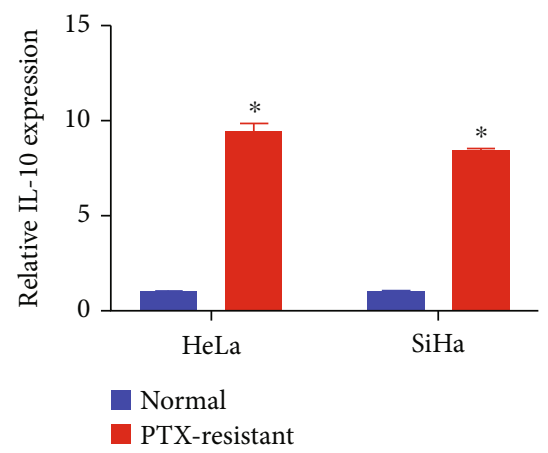

(f)

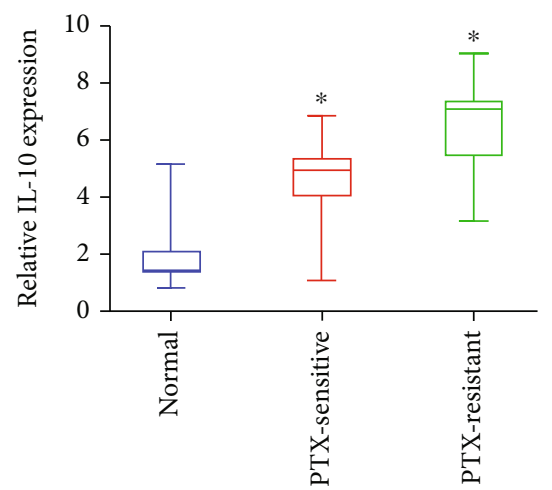

(h)

FIgUre 4: Continued. 


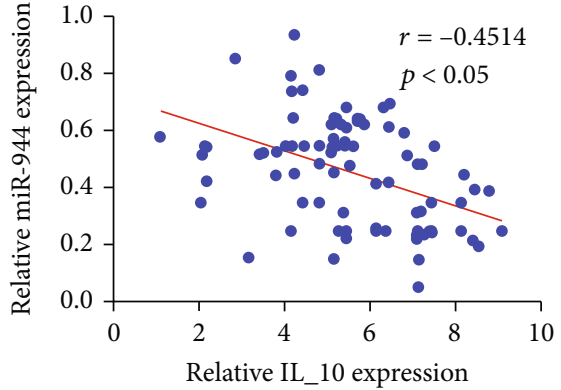

(i)

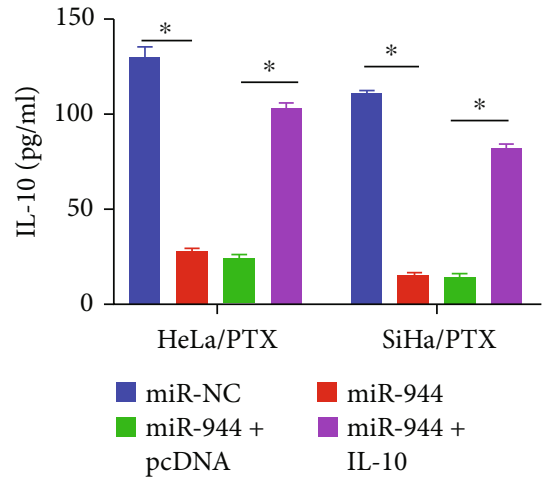

(k)

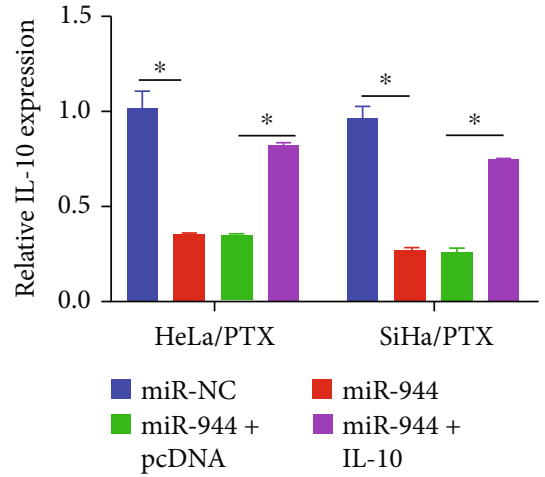

(j)

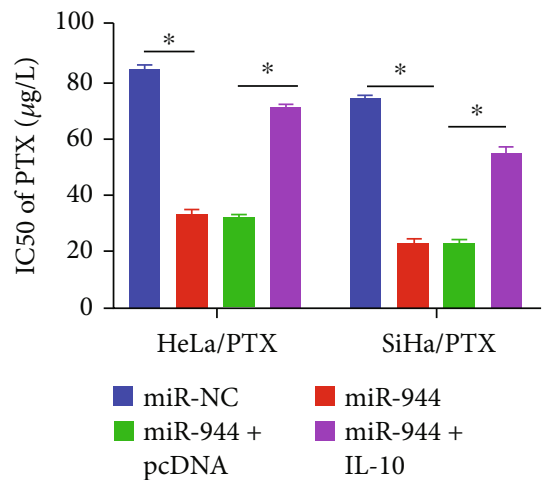

(1)

Figure 4: The targeting of IL-10 in PTX-resistant CC cells results in the overexpression of miR-944 and elevates PTX sensitivity. (a, b) The significant interaction sites between miR-944 and IL-10 determined by the DLR assay. (c) miR-944 expression was assessed via qRT-PCR. $(\mathrm{d}, \mathrm{f}, \mathrm{j})$ The data obtained from qRT-PCR analysis to examine the expression level of IL-10. (e, g, k) The ELISA assay data to detect the expression level of IL-10. (h) The qRT-PCR evaluations for IL-10 level in CC tissues. (i) The correlation between IL-10 and miR-944 was estimated by Spearman's correlation coefficient analysis. (l) The $\mathrm{IC}_{50}$ values of PTX in HeLa/PTX and SiHa/PTX cells. ${ }^{*} p<0.05$.

\subsection{An Elevated Expression of miR-944 Improved PTX} Sensitivity in CC Cells (PTX-Resistant) by IL-10 Targeting. IL-10 was identified to be a target gene of miR-944 using starbase 3.0, and their possible interacting sites are shown in Figure 4(a). Then, using a DLR assay, it was discovered that in miR-944 and IL-10-WT cotransfected 293 T cells; luciferase activity was decreased in comparison to miR-NC and IL-10WT cotransfected groups, but variations were not observed in IL-10-MUT groups (Figure 4(b)). To determine the impact of miR-944 on the expression of IL-10, miR-944, and anti-miR944, their controls were transfected. HeLa/PTX and SiHa/ PTX cells were successfully transfected with miR-944 and anti-miR-944, as shown in Figure 4(c). Furthermore, we evaluated that elevated expression of miR-944 considerably lowered the expression of IL-10 in HeLa/PTX and SiHa/PTX cells but attenuation of miR-944 showed apposing results (Figures 4(d) and 4(e)). Furthermore, our findings revealed that IL-10 expression was decreased in $\mathrm{HeLa}$ and $\mathrm{SiHa}$ cells relative to HeLa/PTX and SiHa/PTX cells, as depicted in Figures 4(f) and 4(g). From healthy tissues to tumor tissues (PTX-sensitive) and subsequently to tumor tissues (PTX-resistant), the expression of IL-10 was elevated (Figure 4(h)). The level of IL-10 mRNA in CC tissues was inversely linked with miR-944 expression using Spearman's correlation coefficient analysis, as revealed in Figure 4(i). miR-195-5p transfection significantly reduced IL-10 expression in HeLa/PTX and SiHa/PTX cells, as depicted in Figures $4(\mathrm{j})$ and $4(\mathrm{k})$, whereas the effects were restored by IL-10 transfection. miR-944 elevated expression successfully decreased PTX resistance in HeLa/PTX and $\mathrm{SiHa} / \mathrm{PTX}$ cells using the CCK-8 assay, while elevated IL-10 successfully reversed the results (Figure 4(1)). In other words, elevated expression of miR-944, which targets IL-10, plays a considerable role in PTX sensitivity in CC cells (PTX-resistant).

3.5. circ_ZFR Knockdown Lowered the IL-10 Expression through Sponging miR-944. For further investigation of the relationships between circ_ZFR, miR-944, and IL-10, the transfection of HeLa/PTX and $\mathrm{SiHa} / \mathrm{PTX}$ cells was carried out with si-NC, si-circ_ZFR, si-circ_ZFR+anti-miR-NC, or si-circ_ZFR+anti-miR-944. In HeLa/PTX and SiHa/PTX cells, silencing of circ_ZFR significantly reduced IL-10 expression, as demonstrated in Figures 5(a) and 5(b), while miR-944 attenuation completely reversed the effects. The IL-10 mRNA level in CC tissue was revealed to be positively linked with the expression of circ_ZFR using Spearman's correlation coefficient analysis, as depicted in Figure 5(c). As a result, we concluded that circ_ZFR favorably controlled IL-10 expression in CC cells (PTX-resistant) via sponging miR-944. 


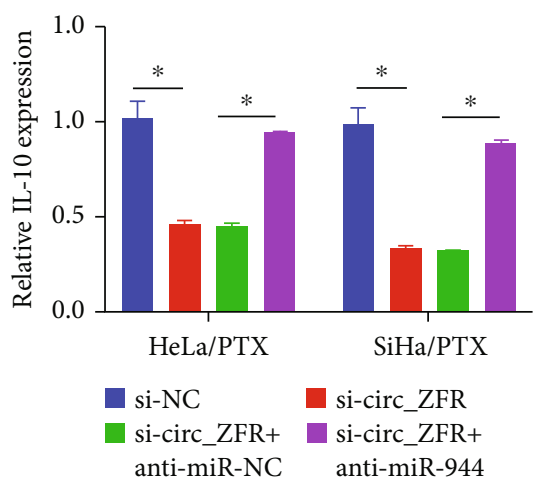

(a)

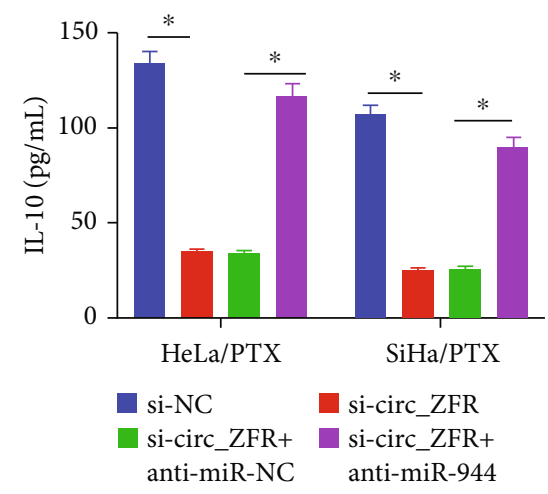

(b)

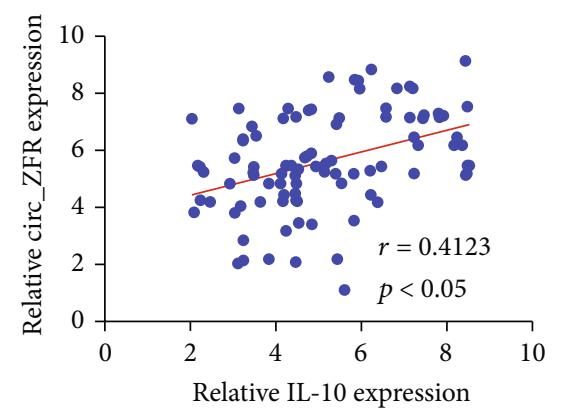

(c)

FIGURE 5: The circ_ZFR knockdown reduced the expression level of IL-10 via miR-944 sponging. (a, b) The data obtained from qRT-PCR and ELISA assay to detect the IL-10 expression. (c) The association between IL-10 and circ_ZFR. ${ }^{*} p<0.05$.

\section{Discussion}

Chemoresistance is a major obstacle in the treatment of human malignancies, including CC. Various circRNAs have been confirmed to have a role in regulating the development of chemoresistance $[22,23]$. The existing study is aimed at seeing what role circ_ZFR plays in modulating CC's PTX sensitivity. As a result of the modulation of the miR-944/ IL-10 axis by circ_ZFR, PTX resistance in CC cells (PTXresistant) was facilitated.

The importance of circRNAs in tumor growth and treatment resistance has steadily drawn researchers' attention in CC. circMTO1, for example, promotes cervical cancer growth and chemoresistance by miR-6893 regulation [21]. hsa-circ 0023404 sponging miR-5047 elevated progression and chemoresistance in cervical cancer via VEGFA and autophagy signaling [24]. The underlined findings revealed that circRNAs performed a variety of roles in CC drug resistance. circ_ZFR has a role in the paclitaxel resistance and development of NSCLC by elevated expression of KPNA4 through sponging miR-195-5p [25]. By modulating the miR-545-3p/CBLL1 axis, circ 0072083 interference suppressed DDP resistance, formation of cell colony, cell cycle, and metastasis and accelerated the apoptotic process in NSCLC cells [26]. In this study, a high level of circ_ZFR was found in chemoresistant CC tissues and cells. In vitro, circ_ZFR deficiency increased PTX sensitivity in CC cells (resistant to PTX). circ_ZFR has a significant contribution to PTX resistance in $\mathrm{CC}$ as a whole.
circ_ZFR was evaluated as the sponge for miR-944, which then boosted IL-6 expression, according to the mechanistic analysis. The miR-944/FZD7 axis attenuated doxorubicin resistance and reduced tumor growth in colorectal cancer when the circular RNA circCSPP1 was knocked down [27]. The obtained results revealed that miR-944 appeared to have a considerable contribution to the development and chemoresistance of CC. Our findings suggested that miR-944 levels were lowered in CC tissues and cells (resistant to PTX). The impact of circ ZFR knockdown on PTX sensitivity may be effectively restored by suppressing miR-944 in CC cells (PTX-resistant). Furthermore, elevated expression of miR-944 boosted the sensitivity of PTX in cells (PTX-resistant); however, these effects might be reversed by an elevated expression of IL-10. Based on the obtained results, miR944 decreased PTX resistance in CC cells (PTX-resistant) by targeting IL-10. In support of our results, AS101 sensitizes tumors to chemotherapy by blocking the tumor IL-10 autocrine loop which supports our findings [28]. Although IL-10 is a target of a variety of miRNAs, including miR-374b-5p [29] and miR-98 [30], we are the first to show an interaction between miR-944 and IL-10 in CC chemoresistance.

In conclusion, the obtained data from the existing study revealed that circ_ZFR enhanced PTX resistance in CC by modulating the miR-944/IL-10 axis, which may have a candidate therapeutic strategy to decrease the resistance of CC to PTX. 


\section{Data Availability}

Due to the nature of this research, participants of this study did not agree for their data to be shared publicly, so supporting data is not available.

\section{Conflicts of Interest}

The authors declare that they have no conflict of interest.

\section{Authors' Contributions}

Haiyan Zhang made substantial contributions to the conception and design of the study and performed the critical revision of the manuscript. Xiaoqian Long and Meiyun Zheng performed the data analysis and interpretation. Xiaoqian Long drafted the manuscript. Youlin Yang performed data curation. Xiaoqian Long performed investigation. Yi Chen and Xiahui Zhang performed software. All co-authors have read the manuscript and approved the final version for submission and publication to this journal.

\section{References}

[1] R. L. Siegel, K. D. Miller, and A. Jemal, "Cancer statistics, 2020," CA: A Cancer Journal for Clinicians, vol. 70, no. 1, pp. 7-30, 2020.

[2] J. Lei, B. Andrae, A. Ploner et al., "Cervical screening and risk of adenosquamous and rare histological types of invasive cervical carcinoma: population based nested case-control study," $B M J$, vol. 365, article 11207, 2019.

[3] L. He, H. Yang, S. Zhou et al., "Synergistic antitumor effect of combined paclitaxel with FEN1 inhibitor in cervical cancer cells," DNA Repair (Amst), vol. 63, pp. 1-9, 2018.

[4] A. C. Panda, I. Grammatikakis, R. Munk, M. Gorospe, and K. Abdelmohsen, "Emerging roles and context of circular RNA," Wiley Interdisciplinary Reviews: RNA, vol. 8, no. 2, 2017.

[5] S. Muller and B. Appel, "In vitro circularization of RNA," RNA Biology, vol. 14, no. 8, pp. 1018-1027, 2017.

[6] F. R. Kulcheski, A. P. Christoff, and R. Margis, "Circular RNAs are miRNA sponges and can be used as a new class of biomarker," Journal of Biotechnology, vol. 238, pp. 42-51, 2016.

[7] S. Meng, H. Zhou, Z. Feng et al., "CircRNA: functions and properties of a novel potential biomarker for cancer," Molecular Cancer, vol. 16, no. 1, p. 94, 2017.

[8] J. M. Nigro, K. R. Cho, E. R. Fearon et al., "Scrambled exons," Cell, vol. 64, no. 3, pp. 607-613, 1991.

[9] Z. Y. Zhang, X. H. Gao, M. Y. Ma, C. L. Zhao, Y. L. Zhang, and S. S. Guo, "CircRNA_101237 promotes NSCLC progression via the miRNA-490-3p/MAPK1 axis," Scientific Reports, vol. 10, no. 1, p. 9024, 2020.

[10] P. Zhou, W. Xie, H. L. Huang et al., "circRNA_100859 functions as an oncogene in colon cancer by sponging the miR217-HIF-1alpha pathway," Aging (Albany NY), vol. 12, no. 13, pp. 13338-13353, 2020.

[11] B. Li, L. Zhu, C. Lu et al., "circNDUFB2 inhibits non-small cell lung cancer progression via destabilizing IGF2BPs and activating anti-tumor immunity," Nature Communications, vol. 12, no. 1 , p. $295,2021$.
[12] M. Stella, L. Falzone, A. Caponnetto et al., "Serum extracellular vesicle-derived circ HIPK3 and circSMARCA5 are two novel diagnostic biomarkers for glioblastoma multiforme," Pharmaceuticals (Basel), vol. 14, no. 7, p. 618, 2021.

[13] X. Y. Mu, Q. Chen, and J. Xie, "Hsa circRNA_104315 increases the malignancy of cervical cancer cells by miR-605-AGO1/ RRM2 pathway," Journal of Biological Regulators and Homeostatic Agents, vol. 34, no. 5, pp. 1771-1777, 2020.

[14] W. Zhang and S. Zhang, "Downregulation of circRNA_ 0000285 suppresses cervical cancer development by regulating miR197-3p-ELK1 axis," Cancer Management and Research, vol. Volume 12, pp. 8663-8674, 2020.

[15] J. Xu, L. Ji, Y. Liang et al., "CircRNA-SORE mediates sorafenib resistance in hepatocellular carcinoma by stabilizing YBX1," Signal Transduction and Targeted Therapy, vol. 5, no. 1, 2020.

[16] Y. Zhao, R. Zheng, J. Chen, and D. Ning, "CircRNA CDRlas/ miR-641/HOXA9 pathway regulated stemness contributes to cisplatin resistance in non-small cell lung cancer (NSCLC)," Cancer Cell International, vol. 20, no. 1, 2020.

[17] M. Dong, P. Li, Y. Xie, Z. Wang, and R. Wang, "CircMYBL2 regulates the resistance of cervical cancer cells to paclitaxel via miR-665-dependent regulation of EGFR," Drug Development Research, vol. 82, no. 8, pp. 1193-1205, 2021.

[18] M. Zhou, Z. Yang, D. Wang, P. Chen, and Y. Zhang, "The circular RNA circZFR phosphorylates Rb promoting cervical cancer progression by regulating the SSBP1/CDK2/cyclin E1 complex," Journal of Experimental \& Clinical Cancer Research, vol. 40, no. 1, 2021.

[19] X. Wang, H. Zhang, H. Yang et al., "Exosome-delivered circRNA promotes glycolysis to induce chemoresistance through the miR-122-PKM2 axis in colorectal cancer," Molecular Oncology, vol. 14, no. 3, pp. 539-555, 2020.

[20] F. W. Zou, S. Z. Yang, W. Y. Li et al., "circRNA_001275 upregulates Wnt7a expression by competitively sponging miR3703p to promote cisplatin resistance in esophageal cancer," International Journal of Oncology, vol. 57, no. 1, pp. 151-160, 2020.

[21] M. Chen, G. Ai, J. Zhou, W. Mao, H. Li, and J. Guo, "circMTO1 promotes tumorigenesis and chemoresistance of cervical cancer via regulating miR-6893," Biomedicine \& Pharmacotherapy, vol. 117, article 109064, 2019.

[22] Y. Sang, B. Chen, X. Song et al., “circRNA_0025202 regulates tamoxifen sensitivity and tumor progression via regulating the miR-182-5p/FOXO3a axis in breast cancer," Molecular Therapy, vol. 27, no. 9, pp. 1638-1652, 2019.

[23] L. Peng, H. Sang, S. Wei et al., "circCUL2 regulates gastric cancer malignant transformation and cisplatin resistance by modulating autophagy activation via miR-142-3p/ROCK2," Molecular Cancer, vol. 19, no. 1, p. 156, 2020.

[24] J. Guo, M. Chen, G. Ai, W. Mao, H. Li, and J. Zhou, "Hsa circ 0023404 enhances cervical cancer metastasis and chemoresistance through VEGFA and autophagy signaling by sponging miR-5047," Biomedicine \& Pharmacotherapy, vol. 115, article 108957, 2019.

[25] J. Li, R. Fan, and H. Xiao, "Circ_ZFR contributes to the paclitaxel resistance and progression of non-small cell lung cancer by upregulating KPNA4 through sponging miR-195-5p," Cancer Cell International, vol. 21, no. 1, p. 15, 2021.

[26] H. Li, F. Liu, and W. Qin, "Circ_0072083 interference enhances growth-inhibiting effects of cisplatin in non-small- 
cell lung cancer cells via miR-545-3p/CBLL1 axis," Cancer Cell International, vol. 20, no. 1, 2020.

[27] L. Xi, Q. Liu, W. Zhang et al., "Circular RNA circCSPP1 knockdown attenuates doxorubicin resistance and suppresses tumor progression of colorectal cancer via miR-944/FZD7 axis," Cancer Cell International, vol. 21, no. 1, 2021.

[28] B. Sredni, M. Weil, G. Khomenok et al., "Ammonium trichloro(dioxoethylene-o, o')tellurate (AS101) sensitizes tumors to chemotherapy by inhibiting the tumor interleukin 10 autocrine loop," Cancer Research, vol. 64, no. 5, pp. 1843-1852, 2004.

[29] Y. Zhang, X. Miao, Z. Zhang et al., "miR-374b-5p is increased in deep vein thrombosis and negatively targets IL-10," Journal of Molecular and Cellular Cardiology, vol. 144, pp. 97-108, 2020.

[30] L. Li, P. Sun, C. Zhang, Z. Li, and W. Zhou, "MiR-98 suppresses the effects of tumor-associated macrophages on promoting migration and invasion of hepatocellular carcinoma cells by regulating IL-10," Biochimie, vol. 150, pp. 23-30, 2018. 